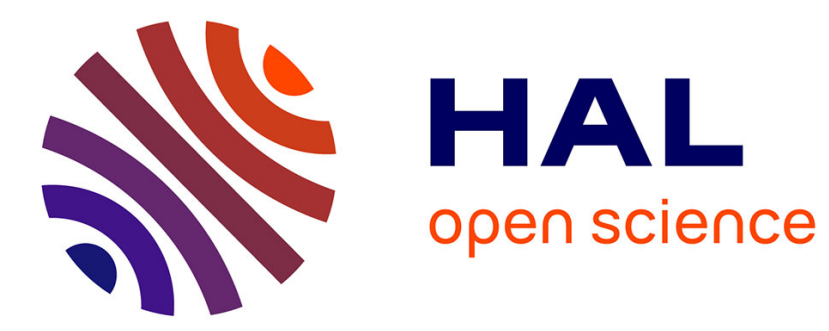

\title{
$250 \mathrm{~W}$ single-crystal fiber Yb:YAG laser
}

Xavier Délen, Stefan Piehler, Julien Didierjean, Nicolas Aubry, Andreas Voss, Marwan Abdou Ahmed, Thomas Graf, François Balembois, Patrick Georges

\section{To cite this version:}

Xavier Délen, Stefan Piehler, Julien Didierjean, Nicolas Aubry, Andreas Voss, et al.. 250 W singlecrystal fiber Yb:YAG laser. Optics Letters, 2012, 37 (14), pp.2898-2900. 10.1364/OL.37.002898 . hal-00854443

\section{HAL Id: hal-00854443 \\ https://hal-iogs.archives-ouvertes.fr/hal-00854443}

Submitted on 27 Aug 2013

HAL is a multi-disciplinary open access archive for the deposit and dissemination of scientific research documents, whether they are published or not. The documents may come from teaching and research institutions in France or abroad, or from public or private research centers.
L'archive ouverte pluridisciplinaire HAL, est destinée au dépôt et à la diffusion de documents scientifiques de niveau recherche, publiés ou non, émanant des établissements d'enseignement et de recherche français ou étrangers, des laboratoires publics ou privés. 


\title{
250 W single-crystal fiber Yb:YAG laser
}

\author{
Xavier Délen, ${ }^{1, *}$ Stefan Piehler, ${ }^{2}$ Julien Didierjean, ${ }^{3}$ Nicolas Aubry, ${ }^{3}$ Andreas Voss, ${ }^{2}$ Marwan Abdou Ahmed, ${ }^{2}$ \\ Thomas Graf, ${ }^{2}$ Francois Balembois, ${ }^{1}$ and Patrick Georges ${ }^{1}$ \\ ${ }^{1}$ Laboratoire Charles Fabry, Institut d'Optique, CNRS, Univ Paris-Sud, 2 Av A. Fresnel, Palaiseau, France \\ ${ }^{2}$ Institut für Strahlwerkzeuge (IFSW), Universität Stuttgart, Pfaffenwaldring 43, 70569 Stuttgart, Germany \\ ${ }^{3}$ Fibercryst SAS, La Doua-Bâtiment l'Atrium, Boulevard Latarjet, F-69616 Villeurbanne Cedex, France \\ ${ }^{*}$ Corresponding author: xavier.delen@institutoptique.fr
}

Received March 30, 2012; revised May 28, 2012; accepted June 1, 2012;

posted June 1, 2012 (Doc. ID 165601); published July 11, 2012

\begin{abstract}
We demonstrate an Yb:YAG single-crystal fiber laser with $251 \mathrm{~W}$ output power in continuous-wave and an optical efficiency of $44 \%$. This performance can be explained by the high overlap between pump and signal beams brought by the pump guiding and by the good thermal management provided by the single-crystal fiber geometry. The oscillator performance with a reflectivity of the output coupler as low as $20 \%$ also shows high potential for power amplification. (c) 2012 Optical Society of America
\end{abstract}

OCIS codes: $140.3615,140.3480$.

High-average power pulsed lasers are widely used in materials processing. Above $100 \mathrm{~W}$ of average power, diode-pumped $\mathrm{Yb}$-doped solid-state lasers implemented in master oscillator power amplifier configuration clearly dominate this field of application with pulse durations ranging from picoseconds to femtoseconds. However, closer investigations indicate that different technologies can be used, each having individual advantages and drawbacks. Optical fiber lasers have proven to be very efficient and to enable high output powers in the continuous-wave regime with up to several $\mathrm{kW}$ of output power in fundamental mode operation. However, due to the strong signal confinement, their use is limited by the damage threshold of the facets and nonlinear effects such as stimulated Raman scattering, self-phase modulation, and self-focusing in the pulsed regime [1]. The thin-disk technology does not exhibit this limitation in the pulsed regime but suffers from relatively low axial gain limited by amplification of spontaneous emission in the transverse direction gain due to the crystal geometry [2], leading to limited energy per pulse and to complex multipass or regenerative amplifier setups. Finally, the slab technology has recently shown clear competitive advantages with over $1 \mathrm{~kW}$ of average output power obtained in the subpicosecond regime and $80 \mathrm{MW}$ peak power [3]. Its drawback is the complexity of the signal beam propagation through the gain medium. Therefore, simple and versatile high power amplifiers for pulsed operation are still an ongoing subject of research.

In this Letter, we show that single-crystal fibers are a promising alternative to these technologies. A singlecrystal fiber is an end-pumped long and thin-rod laser with beam guiding only for the pump radiation. This concept provides a good thermal management thanks to the high thermal conductivity of $\mathrm{Yb}: \mathrm{YAG}$ and the high surface-to-volume ratio offered by the relatively thin and long rod geometry. Moreover, it enables the use of low brightness laser diodes in combination with a long crystal thanks to pump guiding.

Up to now, a maximum of $65 \mathrm{~W}$ of output power in continuous-wave has been demonstrated using an $\mathrm{Yb}$ : YAG single-crystal fiber at a pump power of $200 \mathrm{~W}$ at $940 \mathrm{~nm}$ [4]. This concept has started to reveal its potential as a femtosecond amplifier with a combination of high gain (30) and significant output power (12 W) in a very simple double-pass configuration [5]. In this Letter, we report on an exploration of the step further in terms of power scaling and report on the performance of an Yb:YAG single-crystal fiber oscillator pumped at $600 \mathrm{~W}$ with a fiber coupled laser diode at $940 \mathrm{~nm}$. Furthermore, we discuss the potential of this technology for its use as a power amplifier.

The crystal fiber used in our experiments has a length of $40 \mathrm{~mm}$ and a diameter of $1 \mathrm{~mm}$. With a low doping concentration of $1 \%$, the distribution of the thermal load over the comparatively long rod together with the small transverse dimension of the rod reduces the temperature increase in the gain medium. Finite element analysis indicates that the small distance between the edge of the crystal and the center results in maximum temperature increases as small as $46 \mathrm{~K}$ for $600 \mathrm{~W}$ of incident pump power when using a $1 \mathrm{~mm}$ diameter $40 \mathrm{~mm}$ long crystal with $90 \%$ overall absorption and a $600 \mu \mathrm{m}$ pump spot diameter launched into the crystal. The same temperature difference for a more conventional $3 \mathrm{~mm}$ diameter rod would be more than two times higher and amount to $117 \mathrm{~K}$. Whereas the gradient inside the central volume of the crystal stays the same, the temperature difference between the edge and the center is strongly dependent on the crystal section.

Low doping concentration and small transverse dimensions are not the only parameters to limit the temperature increase: the quality of the thermal contact between the gain medium and its cooled mount may be a key issue as well. As reported in [4], the thermal contact was responsible of $80 \%$ of the total temperature difference between the crystal center and the mount in a single-crystal fiber surrounded by thermal grease in a nonoptimized mount. The Laboratoire Charles Fabry and Fibercryst have worked together to develop an excellent metallic contact in the single-crystal fiber TARANIS modules [ 6 ]. This is discussed in detail in [7]: a temperature difference between the crystal edge and the module of below $5 \mathrm{~K}$ has been measured for $86 \mathrm{~W}$ of incident pump power at $808 \mathrm{~nm}$ on a $0.2 \%$ doped Nd:YAG.

The pump guiding in the single-crystal fiber allows increasing the overlap between the pumped beam and the signal beam in free propagation when compared to 

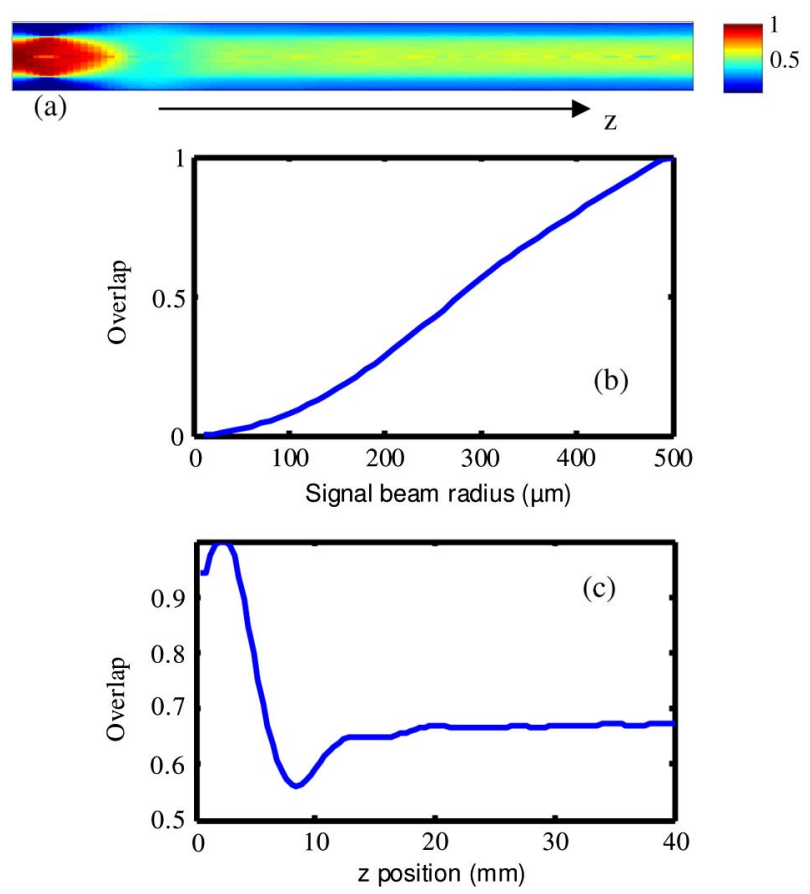

Fig. 1. (Color online) (a) Pump intensity distribution normalized by the maximum of intensity for each $z$ position for a $1 \mathrm{~mm}$ diameter $40 \mathrm{~mm}$ long single-crystal fiber; (b) total geometrical overlap between the pump beam and a flat-top signal beam as a function of the signal beam radius; (c) geometrical overlap between the pump beam and a $700 \mu \mathrm{m}$ diameter flat-top beam as a function of the axial position.

standard bulk technology. Using ray tracing analysis, we obtained the pump intensity distribution in the fiber normalized by the maximum of intensity for each $z$ position. It shows clearly higher intensity in the central area of the crystal brought by the pump guiding in the right part [Fig. 1(a)]. This normalized pump intensity was calculated for an NA of 0.22 and a pump diameter of $600 \mu \mathrm{m}$ at the entrance face of a single-crystal fiber of $1 \mathrm{~mm}$ diameter. The geometrical overlap between a flat-top signal beam and the pump beam is given as a function of the signal beam radius in Fig. 1(b). It shows that up to $69 \%$ of overlap can be obtained for a signal beam diameter of $700 \mu \mathrm{m}$, which covers $70 \%$ of the total rod radius.

Figure 1(c) shows, in each plane along the propagation axis, the overlap between the pump beam and a flattop signal with $700 \mu \mathrm{m}$ diameter corresponding to the experimental conditions described in the following. The overlap first increases until the focal point is reached [see Fig. 1(a)] and starts decreasing afterwards until it reaches a minimum of $56 \%$ at a distance of about $9 \mathrm{~mm}$ from the entrance face of the fiber, corresponding to a position where major part of the pump beam is reflected on the fiber surface. For the last $20 \mathrm{~mm}$ of fiber length, the overlap remains almost constant with a value of $67 \%$. Considering a 90\% overall absorption, we have calculated the overlap increase to be 2.3 times higher than what would be obtained in the same configuration without pump guiding.

In order to explore the potential of the single-crystal fiber in terms of power scalability, a simple two mirror cavity design has been used. As shown in Fig. 2 , the

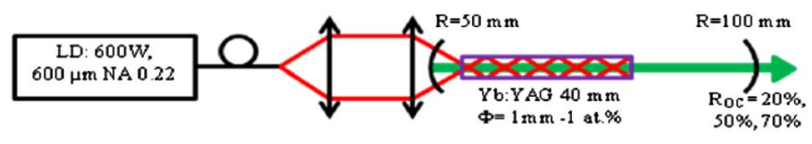

Fig. 2. (Color online) Experimental setup.

single-crystal fiber was placed in between a meniscus with a radius of curvature of $50 \mathrm{~mm}$ and an output coupler with a radius of curvature of $100 \mathrm{~mm}$. The distances "meniscus to crystal" and "crystal to output coupler" were respectively 20 and $85 \mathrm{~mm}$. A fiber coupled diode laser emitting at $940 \mathrm{~nm}$ with $600 \mu \mathrm{m}$ core diameter, an NA of 0.22 , and a maximum output power of $600 \mathrm{~W}$ was used for optical pumping. The fiber output was imaged onto the crystal with a 1:1 magnification ratio using two aspheric lenses of $50 \mathrm{~mm}$ focal length. The TARANIS module provided by Fibercryst contained a $40 \mathrm{~mm}$ long and $1 \mathrm{~mm}$ diameter Yb:YAG single-crystal fiber (1\% doping) placed in a $3 \times 40 \times 70 \mathrm{~mm}^{3}$ copper block. Both crystal faces had an antireflective coating with $0.2 \%$ reflectivity. With a pump absorption around $90 \%$, reabsorption losses at the laser wavelength have a negligible impact since the end of the crystal reaches transparency for an incident pump power around $100 \mathrm{~W}$. Thermal bonding between the crystal and the copper block was ensured by a metallic contact. We estimate that the heat transfer coefficient is around $5 \mathrm{~W} / \mathrm{cm}^{2} / \mathrm{K}$, which is approximately five times better than what can usually be obtained using an indium foil. Mechanical stresses induced by the bonding were sufficiently low to maintain a depolarization below $0.4 \%$. Two copper microchannel plates and carbon foils were used to cool the module. The mounts of the aspheric lenses and the mirrors were water cooled in order to limit the temperature increase of the optics due to possible residual absorption. A dichroic mirror was used to filter out the residual pump power for the output power measurement.

The output power as a function of the incident pump power was measured for three different output couplers having reflectivities of $20 \%, 50 \%$, and $70 \%$. A maximum output power of $251 \mathrm{~W}$ was measured for an output mirror reflecting $70 \%$ and at an incident pump power of $570 \mathrm{~W}$ resulting in an optical efficiency of $44 \%$. The slope efficiency was measured to reach $53 \%$. To the best of our knowledge, this is the highest efficiency ever measured in a single-crystal fiber laser. As shown in Fig. $\underline{3}$, no rollover is visible on the efficiency curve, showing that further power scaling should be possible. Finite element analysis indicates that the pump power limit before fracture should be at $1 \mathrm{~kW}$ for one pumped face.

The beam profile at full pump power is shown in Fig. 3 . We measured a beam quality factor $\mathrm{M}^{2}$ of about 15 . This high value can be explained by two factors. Firstly, due to the lower brightness of the pump beam, no soft aperture mode filtering occurs, as observed previously [4]. Secondly, the cavity design was not fully optimized and we believe that the use of a larger resonant fundamental mode would result in an improvement of the beam quality.

The lowest reflecting mirror $(\operatorname{Roc}=20 \%)$ can give an idea of the potential of the gain medium as power amplifier. Neglecting passive losses, laser oscillation with this mirror means that the active medium provides a 


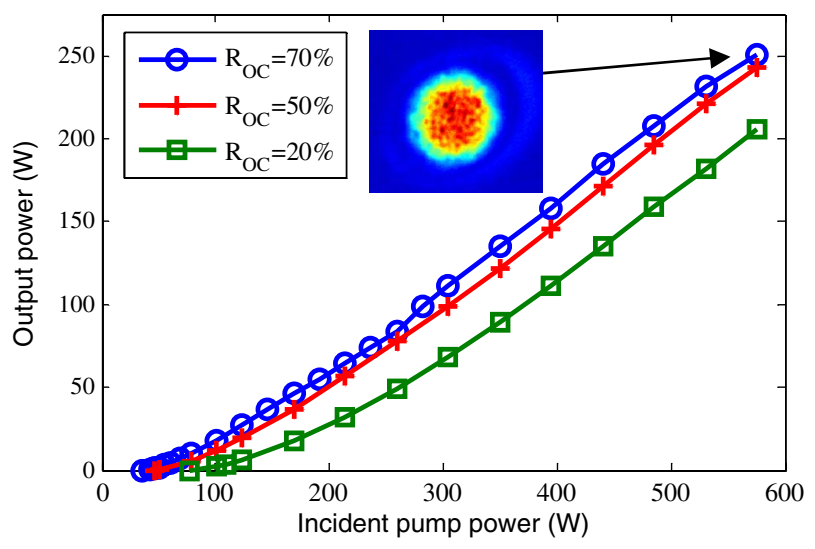

Fig. 3. (Color online) Efficiency curves.

saturated round-trip gain of 5. At maximum pump power, the output power of $200 \mathrm{~W}$ (Fig. 3) means that the gain medium amplifies the intracavity beam from $50 \mathrm{~W}$ (the fraction of power reflected by the $20 \%$ output coupler) to $250 \mathrm{~W}$ in one round-trip. This reveals the high potential of single-crystal fiber technology providing both high gain and significant extraction efficiency for power amplifier design. In order to study the impact of the intensity profile on extraction efficiency, we modelized our singlecrystal fiber amplifier using a similar method to what is described in [8]. Two cases were considered for the signal: a $700 \mu \mathrm{m}$ diameter flat-top beam and a $500 \mu \mathrm{m}$ Gaussian beam. For a pump power of $600 \mathrm{~W}$ and an input signal of $50 \mathrm{~W}$, we found an output power of $258 \mathrm{~W}$ for the flat-top beam and $216 \mathrm{~W}$ for the Gaussian beam. This small difference is due to high saturation of the gain in the case of power amplification. This shows that good extraction efficiency can be expected even for high quality signal beams.
In conclusion, we have demonstrated the potential of the single-crystal fiber technology for the high power regime with more than $250 \mathrm{~W}$ of output power obtained out of an Yb:YAG continuous-wave laser. To our knowledge, this is the present power record, exceeding previous results reported on single-crystal fibers by a factor of 4 . The optical efficiency of up to $44 \%$ and a slope efficiency of $53 \%$ represent the highest efficiency ever achieved with a single-crystal fiber laser. The analysis of the performance obtained with a low reflecting output coupler indicates that a power amplifier boosting power from $50 \mathrm{~W}$ to $250 \mathrm{~W}$ in a two-pass configuration should be feasible with this Yb:YAG single-crystal fiber. This proves that the way is open towards simple and efficient power amplifiers in the "100 W class" for Yb:YAG single-crystal fibers.

Xavier Délen acknowledges the French DGA for the funding of his Ph.D.

\section{References}

1. D. J. Richardson, J. Nilsson, and W. A. Clarkson, J. Opt. Soc. Am. B 27, B63 (2010).

2. D. Blázquez-Sánchez, B. Weichelt, A. Austerschulte, A. Voss, Th. Graf, A. Killi, H. C. Eckstein, M. Stumpf, A. L. Matthes, and U. D. Zeitner, Opt. Lett. 36, 799 (2011).

3. P. Russbueldt, T. Mans, J. Weitenberg, H. D. Hoffmann, and R. Poprawe, Opt. Lett. 35, 4169 (2010).

4. D. Sangla, I. Martial, N. Aubry, J. Didierjean, D. Perrodin, F. Balembois, K. Lebbou, A. Brenier, P. Georges, O. Tillement, and J. M. Fourmigue, Appl. Phys. B 97, 263 (2009).

5. Y. Zaouter, I. Martial, N. Aubry, J. Didierjean, C. Hönninger, E. Mottay, F. Druon, P. Georges, and F. Balembois, Opt. Lett. 36, 748 (2011).

6. www.fibercryst.com.

7. X. Délen, I. Martial, J. Didierjean, N. Aubry, D. Sangla, F. Balembois, and P. Georges, Appl. Phys. B 104, 1 (2011).

8. F. Balembois, M. Castaing, E. Herault, and P. Georges, Laser Photon. Rev. 5, 659 (2011). 